\title{
Assignment Reductions in Inconsistent Ordered Intuition Fuzzy Infor- mation Decision Tables
}

\author{
$\mathrm{Mu} \mathrm{Ke}^{1,{ }^{*}}, \mathrm{Li} \mathrm{Chunli}^{1}$ and Li Sheng ${ }^{2}$ \\ ${ }^{I}$ School of Mathematics and Statistics, Zhoukou Normal University, Zhoukou, Henan, 466000, China \\ ${ }^{2}$ Department of Public Security, Railway Police Collge, Zhengzhou, Henan, 450002, China
}

\begin{abstract}
In the real-world, most information systems are based on dominance relations and may be inconsistent. Moreover, taking the imprecise evaluations in the description of objects into account, intuition fuzzy information systems are introduced to handle with this problem. In this paper, attribute reductions are discussed in inconsistent ordered intuition fuzzy information decision tables. The concept of assignment reductions is proposed which meet different requirements of the existed reductions. Practical approach to compute this kind of reductions is presented by introducing of discernable matrix and discernable function. Moreover, a toy example is employed to illustrate the feasibility of the approach.
\end{abstract}

Keywords: Allocation reductions, dominance-based rough set approach, dominance relations, intuition fuzzy information systems.

\section{INTRODUCTION}

Fuzzy set theory [1] has developed rapidly as computing tool for dealing with uncertainty and imprecision problems, since the elements membership degree was first introduced by Professor Zadeh. Atanassov proposed intuitionistic fuzzy sets [2] concept, because it takes into account the three aspects of the membership degree, non-membership degree and hesitation degree, it is superior to Zadeh fuzzy sets in the area of tackling ambiguity and uncertainty with flexibility and practicality, which is widely used in practical problems [3].

Professor Pawlak proposed rough sets concept [4], it has become a new mathematical tool to deal with ambiguity and uncertainty of knowledge. In recent years, rough set theory has been successfully applied in machine learning, data mining, pattern recognition, and other fields [5]. Attribute reduction is one of the core problems of rough set theory, and the main idea is to keep the classification ability as the premise, and remove unnecessary attributes to make knowledge a simplified by attribute reduction, without losing the basic information $[5,6]$.

Classical rough set theory is based on the equivalence relation. However, in practical problems, many information systems are not based on equivalence relation but on dominance relations (such as product quality, market share, debt ratio, etc.). Therefore, Greco proposed a new rough set theory based on dominance relation $[7,8]$. The knowledge granularity of this theory is constructed through dominance relation of condition attributes sets and decision attributes. In recent years, most research focused on the promotion of information systems based on the dominance relations into general information systems, such as random information systems [9], interval-valued information systems [10], fuzzy objective information systems [11, 12].

Because of the hesitation when evaluating the decision attributes values, the error of the measurement and observation, lack of condition attributes which is associated with decision attribute values and instability decision of information system, the inconsistent decision information system may be caused. In order to deal with inconsistent decision information system, Slezak made possible reduction [13]. Zhang Wenxiu proposed possible reduction's equivalent definition-assignment reduction [14].

The authors suggest a relative reduction of intuition fuzzy decision information system based on dominance relations in [15]. Incomplete intuitionist fuzzy attribute reduction of information systems is researched in [16], the distribution reduction and the largest distribution reduction of inconsistencies intuition fuzzy decision information system got further discussion in $[17,18]$.

This article will continue to discuss the reduction of inconsistency intuitionist fuzzy decision information systems, and propose the concept of assignment reduction, obtain a judging theorem of assignment reduction and specific method for solving all assignment reduction through identification matrix, which further enriches the rough set theory

\section{BASIC CONCEPT}

Compared to Zadeh fuzzy sets, intuitionist fuzzy set theory gives the elements membership in the domain and nonmembership both. 
Definition 1 [2], intuitive fuzzy set is (1) in U domain

$A=\left\{\left(x, \mu_{A}(x), \gamma_{A}(x)\right) \mid x \in U\right\}$

$\mu_{A}(x)$ is the membership degree that $\mathrm{x}$ belongs to $\mathrm{A}$, $\gamma_{A}(x)$ is membership degree that $\mathrm{x}$ does not belongs to A, which meet $0 \leq \mu_{A}(x)+\gamma_{A}(x) \leq 1, \forall x \in U$, $1-\mu_{A}(x)-\gamma_{A}(x)$ is the hesitation degree or uncertainty degree of A.

Definition $2 L^{*}=\left\{\left(x_{1}, x_{2}\right) \in[0,1] \times[0,1] \mid 0 \leq x_{1}+x_{2} \leq 1\right\}$, $\leq_{L^{*}}$ of $L^{*}$ is as below:

$\left(x_{1}, x_{2}\right) \leq_{L^{*}}\left(y_{1}, y_{2}\right) \Leftrightarrow x_{1} \leq y_{1}, x_{2} \geq y_{2}$

From the definition, order relation $\leq_{L^{*}}$ is a partial order relation (which is reflexive, anti-symmetric, transitive), and $\left(L^{*}, \leq_{L^{*}}\right)$ constitutes a complete lattice, greatest element and smallest element are $1_{L^{*}}=(1,0) 0_{L^{*}}=(0,1)$ respectively.

Definition 3 [15], $(U, C \bigcup\{d\}, V, f)$ is intuition fuzzy decision information systems, and $U$ is finite non-empty objects set; $\mathrm{C}$ is finite non-empty condition attribute set; $\mathrm{d}$ is decision attribute and $C \bigcap\{d\}=\varnothing ; \mathrm{V}$ is the attribute value range; $\mathrm{f}$ mapping is object attribute values, which is $U=\left\{x_{1}, x_{2}, \ldots, x_{n}\right\} ; C=\left\{a_{1}, a_{2}, \ldots, a_{s}\right\} ; V=\left(\bigcup_{a \in C} V_{a}\right) \cup V_{d}$, $V_{a}$ is value range of a, Where each element are intuition fuzzy numbers; $f: U \times C \bigcup\{d\} \rightarrow V$, and $f(x, a) \in V_{a}$, which means; $f(x, d) \in\{1,2, \mathrm{~L}, m\}$ is single-valued ordered real value.

If $\gamma_{a}(x)=1-\mu_{a}(x), \forall x \in U, \forall a \in C$, the decision information system will degrade to the general of fuzzy decision Information systems.

Definition 4 [15], supposing that $(U, C \bigcup\{d\}, V, f)$ is intuition decision information system, for any $A \subseteq C$, there $\operatorname{are}(3) \operatorname{and}(4)$

$$
\begin{aligned}
& R_{A}^{\geq}=\left\{(x, y) \mid f(x, a) \geq_{L^{*}} f(y, a), \forall a \in A\right\} \\
& R_{d}^{\geq}=\{(x, y) \mid f(x, d) \geq f(y, d)\}
\end{aligned}
$$

$R_{A}^{\geq}$and $R_{d}^{\geq}$are dominance relations of intuition fuzzy decision information system, which is defined in the condition attribute set $\mathrm{A}$ and decision attribute $\mathrm{d}$, and it called order intuition fuzzy decision information system

In this paper, all the intuition fuzzy decision information systems are order intuition fuzzy decision information system. (5)

The condition attributes " $\mathrm{A}$ " which is dominant to $\mathrm{x}$ is

$[x]_{A}^{\geq}=\left\{y \in U \mid(y, x) \in R_{A}^{\geq}\right\}$

The condition attributes $A$ which is dominant by $x$ is (6)

$[x]_{A}^{\leq}=\left\{y \in U \mid(x, y) \in R_{A}^{\geq}\right\}$

In addition, for $U / R_{d}^{\geq}=\left\{D_{i}^{\geq} \mid i=1,2, \cdots, m\right\}$, $D_{i}^{\geq}=\{x \in U \mid f(x, d) \geq i\}$. Dominance relations, such as $R_{A}^{\geq}$, $[x]_{A}^{\geq}$and $[x]_{A}^{\leq}$, are in references[15-17].

Definition 5 [15], supposing that $(U, C \cup\{d\}, V, f)$ is intuition decision information system, if $R_{C}^{\geq} \subseteq R_{d}^{\geq}$, this decision information system is consistent.

More directly, intuition fuzzy decision information system is inconsistent, if there are elements taking dominant relationship in condition attribute set $\mathrm{C}$, but there is no dominant relationship in the decision attribute.

Example 1 [15]. Table 1 is intuition fuzzy decision information system, and $U=\left\{x_{1}, x_{2}, \cdots, x_{8}\right\}, C=\left\{a_{1}, a_{2}, \cdots, a_{5}\right\}$ are condition attribute set, $\mathrm{d}$ is decision attribute.

From the definition above

$R_{C}^{\geq}=\left\{\left(x_{1}, x_{1}\right),\left(x_{1}, x_{4}\right),\left(x_{2}, x_{2}\right),\left(x_{2}, x_{3}\right),\left(x_{2}, x_{4}\right),\left(x_{3}, x_{3}\right),\left(x_{3}, x_{4}\right)\right.$, $\left.\left(x_{4}, x_{4}\right),\left(x_{5}, x_{5}\right),\left(x_{6}, x_{6}\right),\left(x_{7}, x_{4}\right),\left(x_{7}, x_{7}\right),\left(x_{8}, x_{3}\right),\left(x_{8}, x_{4}\right),\left(x_{8}, x_{8}\right)\right\}$

And $\left(x_{1}, x_{4}\right),\left(x_{3}, x_{4}\right),\left(x_{7}, x_{4}\right) \in R_{C}^{2}$, but $\left(x_{1}, x_{4}\right),\left(x_{3}, x_{4}\right),\left(x_{7}, x_{4}\right) \notin$ $R_{d}^{\geq}$, so $R_{C}^{\geq} \not \subset R_{d}^{2}$, which means this decision information system is not consistent.

\section{ASSIGNMENT REDUCTION OF INCONSISTENT ORDER INTUITION FUZZY DECISION INFOR- MATION SYSTEM}

Supposing that $(U, C \mathrm{U}\{d\}, V, f)$ is inconsistent intuition fuzzy decision information system, for any $A \subseteq C$, there is (7)

$\delta_{A}(x)=\left\{D_{i}^{\geq} \mid[x]_{A}^{\geq} \cap D_{i}^{\geq} \neq \varnothing\right\}$

$\delta_{A}(x)$ is treated as assignment function of condition attributes set $\mathrm{A}$ in domain $\mathrm{U}$

Example 2 (based on example 1). Calculating distribution function on condition attributes of inconsistent intuitive fuzzy decision information system in Table $\mathbf{1}$,

The result is

$\left[x_{i}\right]_{C}^{2}=\left\{x_{i}\right\}, i=1,2,5,6,7,8$;

$\left[x_{3}\right]_{C}^{2}=\left\{x_{2}, x_{3}, x_{8}\right\} ;$ 
Table 1. Inconsistent intuitionist fuzzy sets decision information system.

\begin{tabular}{|c|c|c|c|c|c|}
\hline $\mathbf{U}$ & $\mathbf{a}_{1}$ & $\mathbf{a}_{2}$ & $\mathbf{a}_{3}$ & $\mathbf{a}_{4}$ & $\mathbf{a}_{5}$ \\
\hline \hline $\mathrm{x}_{1}$ & $(0.5,0.4)$ & $(0.5,0.0)$ & $(0.5,0.4)$ & $(0.5,0.4)$ & $(0.5,0.4)$ \\
\hline $\mathrm{x}_{2}$ & $(0.7,0.0)$ & $(0.7,0.1)$ & $(0.6,0.1)$ & $(0.6,0.2)$ & $(0.6,0.1)$ \\
\hline $\mathrm{x}_{3}$ & $(0.6,0.4)$ & $(0.4,0.4)$ & $(0.6,0.1)$ & $(0.4,0.4)$ & $(0.4,0.5)$ \\
\hline $\mathrm{x}_{4}$ & $(0.3,0.6)$ & $(0.3,0.6)$ & $(0.3,0.7)$ & $(0.2,0.2)$ & $(0.3,0.6)$ \\
\hline $\mathrm{x}_{5}$ & $(0.2,0.0)$ & $(0.7,0.0)$ & $(0.6,0.1)$ & $(0.2,0.7)$ \\
\hline $\mathrm{x}_{6}$ & $(0.5,0.3)$ & $(0.5,0.0)$ & $(0.6,0.2)$ & $(0.2,0.0)$ & $(0.6,0.3)$ \\
\hline $\mathrm{x}_{7}$ & $(0.5,0.4)$ & $(0.6,0.4)$ & $(0.4,0.0)$ & $(0.4,0.1)$ & $(0.5,0.4)$ \\
\hline $\mathrm{x}_{8}$ & $(0.7,0.2)$ & $(0.5,0.2)$ & $(0.6,0.0)$ & $(0.6,0.0)$ & $(0.6,0.1)$ \\
\hline
\end{tabular}

$\left[x_{4}\right]_{C}^{\geq}=\left\{x_{1}, x_{2}, x_{3}, x_{4}, x_{7}, x_{8}\right\}_{\circ}$

Thus

$\delta_{C}\left(x_{1}\right)=\delta_{C}\left(x_{7}\right)=\left\{D_{1}^{\geq}, D_{2}^{\geq}\right\}$

$\delta_{C}\left(x_{2}\right)=\delta_{C}\left(x_{3}\right)=\delta_{C}\left(x_{4}\right)=\delta_{C}\left(x_{8}\right)=\left\{D_{1}^{\geq}, D_{2}^{\geq}, D_{3}^{\geq}\right\} ;$

$\delta_{C}\left(x_{5}\right)=\delta_{C}\left(x_{6}\right)=\left\{D_{1}^{2}\right\}$ 。

Property 1 supposing that $(U, C \bigcup\{d\}, V, f)$ is inconsistent intuition fuzzy decision information system,

(1) if $B \subseteq A \subseteq C$, for any $x \in U$, there is $\delta_{A}(x) \subseteq \delta_{B}(x)$

(2) For any $x, y \in U$, if $[x]_{A}^{\geq} \subseteq[y]_{A}^{\geq}$, then $\delta_{A}(x) \subseteq \delta_{A}(y)$

Proving (1) for any $x \in U$, if $B \subseteq A$, then $[x]_{A}^{\geq} \subseteq[x]_{B}^{\geq}$. Then for any $D_{i}^{\geq} \in \delta_{A}(x)$, there is $[x]_{A}^{\geq} \cap D_{i}^{\geq} \neq \varnothing$ and $[x]_{B}^{\geq} \cap D_{i}^{2} \neq \varnothing, D_{i}^{2} \in \delta_{B}(x)$. Thus $\delta_{A}(x) \subseteq \delta_{B}(x)$ is proved

Definition 6. Suppose that $(U, C \cup\{d\}, V, f)$ is inconsistent intuition fuzzy decision information system. For any $x \in U$, there is $\delta_{B}(x)=\delta_{C}(x)$, then $\mathrm{B}$ is treated as assignment coordination set. For further step, If any proper subset of $\mathrm{B}$ are not assigned to coordinate set, then B is Assignment Reduction

Example 3 (based on example 1) Verify that $B=\left\{a_{2}, a_{3}\right\}$ is assignment reduction of inconsistent intuition fuzzy decision information system given in Table $\mathbf{1}$.

By calculating,

$\left[x_{1}\right]_{B}^{\geq}=\left\{x_{1}, x_{5}, x_{6}\right\}$

$\left[x_{2}\right]_{B}^{\geq}=\left\{x_{2}, x_{5}\right\} ;$

$\left[x_{3}\right]_{B}^{\geq}=\left\{x_{2}, x_{3}, x_{5}, x_{8}\right\}$
$\left[x_{4}\right]_{B}^{\geq}=U$;

$\left[x_{i}\right]_{B}^{\geq}=\left\{x_{i}\right\}, i=5,7,8$;

$\left[x_{6}\right]_{B}^{\geq}=\left\{x_{5}, x_{6}\right\}$ 。

Thus $\delta_{B}(x)=\delta_{C}(x), \forall x \in U$, which means that $\mathrm{B}$ is the assignment coordination set。

If $B_{1}=\left\{a_{2}\right\} \quad, \quad$ then $\left[x_{6}\right]_{B_{1}}^{\geq}=\left\{x_{1}, x_{5}, x_{6}\right\}$, $\delta_{B_{1}}\left(x_{6}\right)=\left\{D_{1}^{\geq}, D_{2}^{\geq}\right\} \neq \delta_{C}\left(x_{6}\right)$ 。

If $B_{2}=\left\{a_{3}\right\} \quad, \quad$ then $\left[x_{7}\right]_{B_{2}}^{\geq}=\left\{x_{7}, x_{8}\right\}$, $\delta_{B_{2}}\left(x_{7}\right)=\left\{D_{1}^{\geq}, D_{2}^{\geq}, D_{3}^{\geq}\right\} \neq \delta_{C}\left(x_{7}\right)$ 。

By summing all the results above, it proved that any nonempty subsets are not assigned coordination set, so B is assignment reduction of decision information system.

Then give specific calculation method for solving assignment reduction of inconsistent intuition fuzzy decision information system. First, there is the following decision theorem

Theorem 1. Supposing that $(U, C \bigcup\{d\}, V, f)$ is inconsistent intuition fuzzy decision information system, then B is assignment coordination set $\Leftrightarrow$ For any $x, y \in U$, if $\delta_{C}(x) \not \subset \delta_{C}(y)$, then $[x]_{B}^{\geq} \not \subset[y]_{B}^{\geq}$

Proving $" \Rightarrow$ " (reduction to absurdity) for any $x, y \in U$, if $\delta_{C}(x) \not \subset \delta_{C}(y)$, then $[x]_{B}^{\geq} \subseteq[y]_{B}^{\geq}$and form propertyl(2), there is $\delta_{B}(x) \subseteq \delta_{B}(y)$. But $\mathrm{B}$ is assignment coordination set, so $\delta_{B}(x)=\delta_{C}(x), \forall x \in U$, then $\delta_{C}(x) \subseteq \delta_{C}(y)$ is conflicting with $\delta_{C}(x) \not \subset \delta_{C}(y)$, thus there is $[x]_{B}^{\geq} \not \subset[y]_{B}^{\geq}$.

$" \Leftarrow "$ for $x, y \in U$, when $\delta_{C}(x) \not \subset \delta_{C}(y)$, there is $[x]_{B}^{\geq} \not \subset[y]_{B}^{\geq}$. Thus if $[x]_{B}^{\geq} \subseteq[y]_{B}^{\geq}$, then $\delta_{C}(x) \subseteq \delta_{C}(y)$ 
Table 2. Assigned discernable matrix.

\begin{tabular}{|c|c|c|c|c|}
\hline $\mathbf{D}_{\delta}$ & $\boldsymbol{x}_{\mathbf{1}}$ & $\boldsymbol{x}_{\mathbf{5}}$ & $\boldsymbol{x}_{\mathbf{6}}$ & $\boldsymbol{x}_{7}$ \\
\hline \hline $\boldsymbol{x}_{\mathbf{1}}$ & & $a_{1}, a_{2}, a_{3}, a_{4}$ & $a_{1}, a_{3}, a_{4}, a_{5}$ & $a_{3}, a_{4}$ \\
\hline $\boldsymbol{x}_{2}$ & $a_{2}$ & $a_{2}$ & $a_{2}, a_{4}$ & $a_{2}, a_{3}, a_{4}, a_{5}$ \\
\hline$x_{3}$ & $a_{2}, a_{4}, a_{5}$ & $a_{1}, a_{2}, a_{4}, a_{5}$ & $C$ \\
\hline$x_{4}$ & $C$ & $a_{1}, a_{2}, a_{4}$ & $C$ \\
\hline$x_{7}$ & & $a_{1}, a_{2}, a_{3}, a_{4}$ & $C$ \\
\hline$x_{8}$ & $a_{2}$ & $a_{1}, a_{2}, a_{3}$ & $a_{2}$ & $a_{2}$ \\
\hline
\end{tabular}

For any $D_{i}^{\geq} \in \delta_{B}(x)$, there is $[x]_{B}^{2} \cap D_{i}^{\geq} \neq \varnothing$, supposing $y \in[x]_{B}^{\geq} \cap D_{i}^{\geq}$, then $y \in D_{i}^{\geq}$and $y \in[x]_{B}^{\geq}$, which is $[y]_{B}^{\geq} \subseteq[x]_{B}^{\geq}$. So $\delta_{C}(y) \subseteq \delta_{C}(x)$. In addition $y \in[y]_{C}^{\geq}$, which is $D_{i}^{\geq} \cap[y]_{C}^{\geq} \neq \varnothing$, so $D_{i}^{\geq} \in \delta_{C}(y)$ and $D_{i}^{\geq} \in \delta_{C}(x)$ further, then $\delta_{B}(x) \subseteq \delta_{C}(x)$.

On the other hand, from propertyl(1) there is $\delta_{C}(x) \subseteq \delta_{B}(x)$, in summary $\delta_{C}(x)=\delta_{B}(x)$, which means B is assignment coordination set.

Definition 7. Supposing that $(U, C \bigcup\{d\}, V, f)$ is inconsistent intuition fuzzy decision information system, and $D_{\delta}^{*}=\left\{(x, y) \mid \delta_{C}(x) \not \subset \delta_{C}(y)\right\}$, define

$D_{\delta}(x, y)=\left\{\begin{array}{lr}\left\{a \in A T \mid(x, y) \notin R_{\{a\}}^{\geq}\right\} & (x, y) \in D_{\delta}^{*} \\ \varnothing, & (x, y) \notin D_{\delta}^{*}\end{array}\right.$

$D_{\delta}(x, y)$ is assigned discernible attribute set of $\mathrm{x}$ and $\mathrm{y}$. The matrix $\mathbf{D}_{\delta}=\left\{D_{\delta}(x, y) \mid x, y \in U\right\}$ is assigned discernable matrix of decision system.

Example 4 (based on example 1), calculating the discernable attribute matrix of inconsistent intuition fuzzy decision information system in Table $\mathbf{1}$.

From example 2, it is known $D_{\delta}^{*}=\left\{\left(x_{1}, x_{5}\right),\left(x_{1}, x_{6}\right),\left(x_{2}, x_{1}\right),\left(x_{2}, x_{5}\right),\left(x_{2}, x_{6}\right), \quad\left(x_{2}, x_{7}\right),\left(x_{3}, x_{1}\right)\right.$, $\left(x_{3}, x_{5}\right),\left(x_{3}, x_{6}\right),\left(x_{3}, x_{7}\right),\left(x_{4}, x_{1}\right),\left(x_{4}, x_{5}\right),\left(x_{4}, x_{6}\right),\left(x_{4}, x_{7}\right),\left(x_{7}, x_{5}\right)$, $\left.\left(x_{7}, x_{6}\right),\left(x_{8}, x_{1}\right),\left(x_{8}, x_{5}\right),\left(x_{8}, x_{6}\right),\left(x_{8}, x_{7}\right)\right\}$, and discernable attribute matrix of decision information system as Table 2.

Theorem 2. Supposing that $(U, C \bigcup\{d\}, V, f)$ is inconsistent intuition fuzzy decision information system, $B \subseteq C$, then $\mathrm{B}$ is assignment coordination set $\Leftrightarrow$ for any $(x, y) \in D_{\delta}^{*}$, there is $B \cap D_{\delta}(x, y) \neq \varnothing$
Proving, $\quad " \Rightarrow "$ for any $(x, y) \in D_{\delta}^{*} \quad$ there is $\delta_{C}(x) \not \subset \delta_{C}(y)$. Form B is assignment coordination set and Theorem1, there is $[x]_{B}^{\geq} \not \subset[y]_{B}^{2}$, so $a \in B$ which makes $(x, y) \notin R_{\{a\}}^{\geq}$, it is $a \in D_{\delta}(x, y)$. Thus $B \cap D_{\delta}(x, y) \neq \varnothing$.

$" \Leftarrow "$ for any $(x, y) \in D_{\delta}^{*}$, which is $\delta_{C}(x) \not \subset \delta_{C}(y)$, there is $B \cap D_{\delta}(x, y) \neq \varnothing$, then $a \in C$ makes $a \in B$, which means $(x, y) \notin R_{\{a\}}^{\geq}$, so $x \notin[y]_{a}^{\geq}$. In addition, there are $x \notin[y]_{B}^{\geq}$and $x \in[x]_{B}^{\geq}$, so $[x]_{B}^{\geq} \not \subset[y]_{B}^{\geq}$. Form Theorem1, B is assignment coordination set.

Definition 8. Supposing that $(U, C U\{d\}, V, f)$ is inconsistent intuition fuzzy decision information system, $\mathbf{D}_{\delta}$ is assigned discernable matrix, there is (9)

$M_{\delta}=\wedge \vee\left\{a \mid a \in D_{\delta}(x, y), x, y \in U\right\}$

$M_{\delta}$ is the assigned discernable function of this decision information system.

Theorem 3. Supposing that $(U, C \bigcup\{d\}, V, f)$ is inconsistent intuition fuzzy decision information system, $M_{\delta}$ is assigned discernable function of this decision information system, $M_{\delta}$ 's minimum disjunctive norm is (10)

$M_{\delta}=\wedge_{k=1}^{t} \vee_{s=1}^{q_{k}}\left(a_{i_{s}}\right)$

If $B_{\delta}=\left\{a_{i_{s}} \mid s=1,2, \cdots, q_{k}\right\}$, then $\left\{B_{\delta} \mid k=1,2, \cdots, t\right\}$ is assignment reduction of this decision information system.

Proving. It is proved by Theorem 2 and minimum disjunctive norm directly.

Example 4 (based on example 1), calculating the assignment reduction of inconsistent intuition fuzzy decision information system in Table 1. 
From example 2 and 4 , the result is as below

$$
M_{\delta}=a_{2} \wedge\left(a_{3} \vee a_{4}\right)=\left(a_{2} \wedge a_{3}\right) \vee\left(a_{2} \wedge a_{4}\right) \text {, thus the two }
$$

assignment reductions are $\left\{a_{2}, a_{3}\right\}$ and $\left\{a_{2}, a_{4}\right\}$.

\section{CONCLUSION}

In this paper, we introduce the concept of assignment reduction based on dominance relations of intuition inconsistent fuzzy decision information system, propose judgment assignment reduction theorem and identification matrix, and take use of the distribution function to calculate assignment reduction of information system, which expand the study based on dominance relations of rough set theory.

\section{CONFLICT OF INTEREST}

The authors confirm that this article content has no conflict of interest.

\section{ACKNOWLEDGEMENTS}

Declared none.

\section{REFERENCES}

[1] L. Zadeh, "A fuzzy sets," Information and Control, vol. 8, no. 3, pp. 338-353, 1965.

[2] K.T. Atanassov, "Intuitionistic fuzzy sets," Fuzzy Sets and Systems, vol. 20 , no. 1, pp. 87-96, 1986.

[3] Z. Xu "Intuitionistic Fuzzy Information Integration Theory and Application," Science Press: Beijing 2008.

[4] Z. Pawlak, "Rough sets," International Journal of Computer and Information Sciences, vol. 11, no. 5, pp. 341-356, 1982.
[5] G. Wang, Rough set Theory and Knowledge Acquisition, Xi'an, Xi'an Jiaotong University Press, 2001.

[6] W. Zhang, W. Wu, L. Zhi, Rough Set Theory and Methods, Science Press, Beijing 2001.

[7] S. Greco, B. Matarazzo, and R. Slowinski, "Rough approximation by dominance relations," International Journal of Intelligent Systems, vol. 17, no. 2, pp. 153-171, 2002.

[8] S. Greco, B. Matarazzo, and R. Slowinski, "Rough sets theory for multicriteria decision analysis," European Journal of Operational Research, vol. 129, no. 1, pp. 1-47, 2001.

[9] X. Qiu, and L. Wei. "Attribute reduction of random information systems based on dominance relation," Computer Engineering and Applications, vol. 47, no. 2, pp. 131-135, 2011.

[10] J. Mao, D. Yao, C. Wang, and T. Wu, "Probability-rough model on $\alpha$-dominance relationship and its application," Computer Engineering and Applications, vol. 48, no. 18, pp. 48-52, 2012.

[11] Y. Zhao, and W. Du, "Attribute reductions in fuzzy objective information systems based on dominance relations," Computer Engineering and Applications, vol. 48, no. 27, pp. 132-135, 2012.

[12] M. Inuiguchi, Y. Yoshioka, and Y. Kusunoki , "Variable-precision dominance-based rough set approach and attribute reduction," International Journal of Approximate Reasoning, vol. 50, no. 8, pp. 1199-1214, 2009.

[13] D. Slezak, "Searching for dynamic reducts in inconsistent decision tables," In: Proc of IPMU'98, Paris, France, vol. 2, pp. 1362-1369, 1998.

[14] W.X. Zhang, J.S. Mi, and W.Z. Wu, "Approaches to knowledge reductions in inconsistent systems," International Journal of Intelligent Systems, vol. 18, no. 9, pp. 989-1000, 2003.

[15] W. Du, B. Hu, and Z. Yan, "Intuition based on dominance relations fuzzy information systems Attribute Reduction," Operations Research and Fuzzy Science, vol. 1, no. 1, pp. 1-5, 2011.

[16] X. Hai, and B. Hu, "Incomplete fuzzy information systems intuition attribute Reduction," Mathematics in Practice and Theory, vol. 42, no. 19, pp. 210-217, 2012.

[17] W. Du, B. Hu, and Z. Yan, "Inconsistent intuitionistic fuzzy reduction decision information systems," Fuzzy Systems and Mathematics, vol. 27, no. 3, pp. 169-175, 2013.

[18] W. Wei, Y. Qi, "Information potential fields navigation in wireless ad-hoc sensor networks," Sensors, pp. 4794-4807; UT WOS:000290947700019, 2011.

(C) Ke et al.; Licensee Bentham Open.

This is an open access article licensed under the terms of the Creative Commons Attribution Non-Commercial License (http://creativecommons.org/licenses/by-nc/3.0/) which permits unrestricted, non-commercial use, distribution and reproduction in any medium, provided the work is properly cited. 